\title{
Robust PID Decentralized Controller Design Using LMI
}

\author{
Danica Rosinová, Vojtech Veselý
}

\begin{abstract}
The new LMI based method for robust stability analysis for linear uncertain system with PID controller is proposed. The general constrained structure of controller matrix is considered appropriate for both output feedback and decentralized control and the respective guaranteed cost control design scheme is presented. The sufficient robust stability condition is developed for extended quadratic performance index including first derivative of the state vector to damp oscillations. The obtained stability condition is formulated for parameter-dependent Lyapunov function.

Keywords: Uncertain systems, Robust stability, Decentralized control, Linear matrix inequalities (LMI), Lyapunov function
\end{abstract}

\section{Introduction}

Robust stability and robust control belong to fundamental problems in control theory and practice; various approaches in this field have been proposed to cope with uncertainties that always appear in real plant ([2];[8];[7];[5];[4]). The development of Linear Matrix Inequality (LMI) computational techniques has brought an efficient tool to solve a large set of convex problems in polynomial time (e.g. [2]). Significant effort has been therefore made to formulate crucial control problems in algebraic way ([12]), so that the numerical LMI solution can be adopted. This approach is advantageously used in solving control problems for linear systems with convex (affine or polytopic) uncertainty domain. However, many important problems in linear control design, such as decentralized control, simultaneous SOF or more generally - structured linear control problems have been proven as NP hard ([1]). Intensive research has been devoted to overcome nonconvexity and transform the nonconvex or NP-hard problem into convex optimisation problem in LMI framework. Various techniques have been developed using inner or outer convex approximation of the respective nonconvex domain. The common tool in both inner and outer approximation is the use of linearization or convexification. In ([6]; [3]) the general convexifying algorithm for the nonconvex function together with potential convexifying functions for both continuous and discrete-time case have been proposed. Linearization approach for continuous and discrete-time system design was independently used in ([13];[11]).

Proportional-integral-derivative (PID) controllers belong to the most popular ones in the industrial world. The derivative part of the controller, however, causes difficulties when uncertainties are considered. In multivariable PID control schemes using LMI developed recently ([14]) the incorporation of the derivative part requires inversion of the respective matrix, which does not allow including uncertainties. The other way to cope with the derivative part is to assume the special case when output and its derivative are state variables, robust PID controller for first and second order SISO systems are proposed for this case in ([7]).

In this paper a state space approach to designing decentralized (multi-loop) PID robust controllers is proposed for linear uncertain system with guaranteed cost with a new quadratic cost function. The major contribution is in considering the derivative part in robust control framework. We adopt the new PID control problem formulation using LMI that is appropriate for polytopic uncertain systems. The robust PID control scheme is proposed for structured control gain matrix, thus enabling decentralized PID control design. In Section 2 the robust control design problem with structured control gain matrix is formulated in general. The robust optimal control design procedure in state space with the extended cost function is proposed in Section 3. The main result-robust PID controller design approach is provided in Section 
4, the developed approach is appropriate for decentralized control structure. In Section 5 the results are illustrated on the examples.

\section{Problem Formulation And Preliminaries}

Consider a linear affine uncertain system:

$$
\begin{gathered}
\delta x(t)=(A+\delta A) x(t)+(B+\delta B) u(t) \\
y(t)=C x(t)
\end{gathered}
$$

where

$$
\begin{aligned}
& \delta x(t)=\dot{x}(t) \text { for continuous - time system } \\
& \delta x(t)=x(t+1) \text { for discrete - time system }
\end{aligned}
$$

$x(t) \in R^{n}, u(t) \in R^{m}, y(t) \in R^{l}$ are state, control and output vectors respectively; $A, B, C$ are known constant matrices of appropriate dimensions corresponding to the nominal system, $\delta A, \delta B$ are matrices of uncertainties of the respective dimensions. The uncertainties are considered to be affine of the form

$$
\delta A=\sum_{j=1}^{p} \varepsilon_{j} \tilde{A}_{j}, \delta B=\sum_{j=1}^{p} \varepsilon_{j} \tilde{B}_{j}
$$

where $\underline{\varepsilon}_{j} \leq \varepsilon_{j} \leq \bar{\varepsilon}_{j}$ are unknown uncertainty parameters; $\tilde{A}_{j}, \tilde{B}_{j}, j=1,2, \ldots, p$ are constant matrices of uncertainties of the respective dimensions and structure. The uncertain system (1), (2) can be equivalently described by a polytopic model given by its vertices

$$
\left\{\left(A_{1}, B_{1}, C\right),\left(A_{2}, B_{2}, C\right), \ldots,\left(A_{N}, B_{N}, C\right)\right\}, N=2^{p} .
$$

The decentralized feedback control law is considered in the form

$$
u(t)=F C x(t)
$$

where $F$ is a matrix corresponding to decentralized controller. The uncertain closed-loop polytopic system is then

$$
\delta x(t)=A_{C}(\alpha) x(t)
$$

where

$$
\begin{aligned}
& A_{C}(\alpha) \in\left\{\sum_{i=1}^{N} \alpha_{i} A_{C i}, \sum_{i=1}^{N} \alpha_{i}=1, \alpha_{i} \geq 0\right\}, \\
& A_{C i}=A_{i}+B_{i} F C .
\end{aligned}
$$

To assess the performance quality a quadratic cost function known from LQ theory is often used. However, in practice the response rate or overshoot are often limited. Therefore we include into the cost function the additional derivative term for state variable to open the possibility to damp the oscillations and limit the response rate.

$$
J_{c}=\int_{0}^{\infty}\left[x(t)^{T} Q x(t)+u(t)^{T} R u(t)+\delta x(t)^{T} S \delta x(t)\right] d t
$$

for a continuous-time and

$$
J_{d}=\sum_{k=0}^{\infty}\left[x(t)^{T} Q x(t)+u(t)^{T} R u(t)+\delta x(t)^{T} S \delta x(t)\right]
$$


for a discrete-time system,

where $Q, S \in R^{n \times n}, R \in R^{m \times m}$ are symmetric positive definite matrices. The concept of guaranteed cost control is used in a standard way: Let there exist a feedback gain matrix $F_{0}$ and a constant $J_{0}$ such that

$$
J \leq J_{0}
$$

holds for the closed loop system (4), (5). Then the respective control (3) is called the guaranteed cost control and the value of $J_{0}$ is the guaranteed cost. The main aim of this paper is to develop a decentralized PID control algorithm that stabilizes the uncertain system (1), with guaranteed cost with respect to the cost function (6).

We start with basic notions concerning Lyapunov stability and convexifying functions. In the following we use $D$-stability concept ([4]) to receive the respective stability conditions in more general form.

Definition 1. (D-stability) Consider the $D$-domain in the complex plain defined as

$$
D=\left\{s \text { is complex number : }\left[\begin{array}{l}
1 \\
s
\end{array}\right]^{*}\left[\begin{array}{ll}
r_{11} & r_{12} \\
r_{12}^{*} & r_{22}
\end{array}\right]\left[\begin{array}{l}
1 \\
s
\end{array}\right]<0\right\} .
$$

The considered linear system (1) is $D$-stable if all its poles lie in the $D$-domain.

(To simplify the reading of formulas we use in the Definition 1 scalar values of the parameters $r_{i j}$, in general the stability domain can be defined using matrix values of parameters $r_{i j}$ with the respective dimensions.) The standard choice of $r_{i j}$ is $r_{11}=0, r_{12}=1, r_{22}=0$ for a continuous-time system; $r_{11}=-1, r_{12}=0, r_{22}=1$ for a discrete-time system. The quadratic D-stability is equivalent to the existence of one Lyapunov function for the whole set that describes the uncertain system model.

Definition 2. (Quadratic $D$ - stability)

The uncertain system (4) is quadratically $D$-stable if and only if there exists a symmetric positive definite matrix $P$ such that

$$
r_{12} P A_{C}(\alpha)+r_{12}^{*} A_{C}^{T}(\alpha) P+r_{11} P+r_{22} A_{C}^{T}(\alpha) P A_{C}(\alpha)<0
$$

Instead of quadratic stability, a robust stability notion is considered based on the parameter dependent Lyapunov function (PDLF) defined as

$$
P(\alpha)=\sum_{i=1}^{N} a_{i} P_{i} \text { where } P_{i}=P_{i}^{T}>0
$$

to obtain less conservative results than using quadratic stability with unique Lyapunov function.

Definition 3. ([5]) System (4) is robustly D-stable in the convex uncertainty domain (5) with parameterdependent Lyapunov function (9) if and only if there exists a matrix $P(\alpha)=P(\alpha)^{T}>0$ such that

$$
\begin{gathered}
r_{12} P(\alpha) A_{C}(\alpha)+r_{12}^{*} A_{C}^{T}(\alpha) P(\alpha)+r_{11} P(\alpha)+ \\
+r_{22} A_{C}^{T}(\alpha) P(\alpha) A_{C}(\alpha)<0
\end{gathered}
$$

for all $\alpha$ such that $A_{C}(\alpha)$ is given by (5).

The sufficient robust $D$-stability condition which can be considered as not too conservative has been proposed in ([9]), recalled in the following lemma.

Lemma 4. If there exist matrices $E \in R^{n x n}, G \in R^{n x n}$ and $N$ symmetric positive definite matrices $P_{i} \in R^{n x n}$ such that for all $i=1, \ldots, N$ :

$$
\left[\begin{array}{cc}
r_{11} P_{i}+A_{C i}^{T} E^{T}+E A_{C i} & r_{12} P_{i}-E+A_{C i}^{T} G \\
r_{12}^{*} P_{i}-E^{T}+G^{T} A_{C i} & r_{22} P_{i}-\left(G+G^{T}\right)
\end{array}\right]<0
$$

then system (4) is robustly D-stable. 
Note that matrices $E$ and $G$ are not restricted to any special form; they were included to relax the conservatism of the sufficient condition. To transform nonconvex problem of structured control (decentralized control in our case) into convex form, the convexifying (linearizing) function can be used ([6]; [3];[11];[13]). The respective potential convexifying function for $X^{-1}$ and $X W X$ has been proposed in the linearizing form:

- The linearization of $X^{-1} \in R^{n x n}$ about the value $X_{k}>0$ is

$$
\Phi\left(X^{-1}, X_{k}\right)=X_{k}^{-1}-X_{k}^{-1}\left(X-X_{k}\right) X_{k}^{-1}
$$

- The linearization of $X W X \in R^{n x n}$ about $X_{k}$ is

$$
\Psi\left(X W X, X_{k}\right)=-X_{k} W X_{k}+X W X_{k}+X_{k} W X
$$

Both functions defined in (12) and (13) meets one of the basic requirements on convexifying function: to be equal to the original nonconvex term if and only if $X_{k}=X$. However, the question how to choose the appropriate nice convexifying function remains still open.

In the sequel, $X>0$ denotes positive definite matrix; * in matrices denotes the respective transposed term to make the matrix symmetric; $I$ denotes identity matrix and 0 denotes zero matrix of the respective dimensions.

\section{Robust Optimal Controller Design}

In this section the new design algorithm for optimal control with guaranteed cost is developed using parameter dependent Lyapunov function and convexifying approach employing iterative procedure. The proposed control design approach uses sufficient stability condition inspired by the result of ([9]). The next theorem provides the new form of robust stability condition for linear uncertain system with guaranteed cost.

Theorem 5. Consider uncertain linear system (1), (2) with static output feedback (3) and cost function (6). The following statements are equivalent:

i) Closed loop system (4) is robustly D-stable with PDLF (9) and guaranteed cost with respect to cost function (6): $J \leq J_{0}=x^{T}(0) P(\alpha) x(0)$.

ii) There exist matrices $P(\alpha)>0$ defined by (9) such that

$$
\begin{aligned}
& r_{12} P(\alpha) A_{C}(\alpha)+r_{12}^{*} A_{C}^{T}(\alpha) P(\alpha)+r_{22} A_{C}^{T}(\alpha) P(\alpha) A_{C}(\alpha)+ \\
& \quad+r_{11} P(\alpha)+Q+C^{T} F^{T} R F C+A_{C}^{T}(\alpha) S A_{C}(\alpha)<0
\end{aligned}
$$

iii) There exist matrices $P(\alpha)>0$ defined by (9) and $H, G$ and $F$ of the respective dimensions such that

$$
\begin{aligned}
& {\left[\begin{array}{c}
r_{11} P(\alpha)+A_{C}^{T}(\alpha) H^{T}+H A_{C}(\alpha)+Q+C^{T} F^{T} R F C \\
r_{12}^{*} P(\alpha)-H^{T}+G^{T} A_{C}(\alpha)
\end{array}\right] .} \\
& {\left[\begin{array}{c}
* \\
r_{22} P(\alpha)-\left(G+G^{T}\right)+S
\end{array}\right]<0}
\end{aligned}
$$

$A_{C i}=\left(A_{i}+B_{i} F C\right)$ denotes the $i-t h$ closed loop system vertex. Matrix $F$ is the guaranteed cost decentralized control gain for the uncertain system (4), (5). 
Proof. For brevity the detail steps of the proof are omitted where standard tools are applied. $(i) \Leftrightarrow(i i)$ : the proof is analogous to that in ([10]). The (ii) $\Rightarrow(\mathrm{i})$ is shown by taking $V(t)=x(t) P(\alpha) x(t)$ as a candidate Lyapunov function for (4) and writing $\delta V(t)$, where

$$
\begin{gathered}
\delta V(t)=\dot{V}(t) \text { for continuous - time system } \\
\delta V(t)=V(t+1)-V(t) \text { for discrete - time system } \\
\delta V(t)=r_{12}^{*} \delta x(t)^{T} P(\alpha) x(t)+r_{12} x(t)^{T} P(\alpha) \delta x(t)+r_{11} x(t)^{T} P(\alpha) x(t)+r_{22} \delta x(t)^{T} P(\alpha) \delta x(t)
\end{gathered}
$$

Substituting for $\delta x$ from (4) to (16) and comparing with (14) provides $D$-stability of the considered system when the latter inequality holds. The guaranteed cost can be proved by summing or integrating both sides of the following inequality for $t$ from 0 to $\infty$ :

$$
\delta V(t)<-x(t)^{T}\left[Q+C^{T} F^{T} R F C+A_{C}^{T}(\alpha) S A_{C}(\alpha)\right] x(t)
$$

The (i) $\Rightarrow$ (ii) can be proved by contradiction. (ii) $\Leftrightarrow(i i i)$ : The proof follows the same steps to the proof of Lemma 1: (iii) $\Rightarrow$ (ii) is proved in standard way multiplying both sides of (15) by the full rank matrix:

$$
\left[\begin{array}{ll}
I & A_{C}^{T}(\alpha)
\end{array}\right]\{\text { l.h.s. }(15)\}\left[\begin{array}{c}
I \\
A_{C}(\alpha)
\end{array}\right]<0 .
$$

(ii) $\Rightarrow$ (iii) follows from applying a Schur complement to (14) rewritten as

$$
\begin{aligned}
& r_{12} P(\alpha) A_{C}(\alpha)+r_{12}^{*} A_{C}^{T}(\alpha) P(\alpha)+Q+C^{T} F^{T} R F C+ \\
& \quad+r_{11} P(\alpha)+A_{C}^{T}(\alpha)\left[r_{22} P(\alpha)+S\right] A_{C}(\alpha)<0
\end{aligned}
$$

Therefore $\left[\begin{array}{ll}X_{11} & X_{12} \\ X_{12}^{T} & X_{22}\end{array}\right]<0$ where

$$
\begin{aligned}
& X_{11}=r_{11} P(\alpha)+r_{12} P(\alpha) A_{C}(\alpha)+r_{12}^{*} A_{C}^{T}(\alpha) P(\alpha)+Q+ \\
& \quad+C^{T} F^{T} R F C \\
& X_{12}=A_{C}^{T}(\alpha)\left[r_{22} P(\alpha)+S\right] \\
& X_{22}=-\left[r_{22} P(\alpha)+S\right]
\end{aligned}
$$

which for $H=r_{12} P(\alpha), G=\left[r_{22} P(\alpha)+S\right]$ gives (15).

The guaranteed cost control design is based on the robust stability condition (15). Since the matrix inequality (15) is not LMI we use the inner approximation for the continuous time system applying linearization formula (13) together with using the respective quadratic forms to obtain LMI formulation, which is then solved by iterative procedure.

\section{PID Robust Controller Design For Continuous-Time Systems}

Control algorithm for PID is considered as

$$
u(t)=K_{P} y(t)+K_{I} \int_{0}^{t} y(t) d t+F_{d} C_{d} \dot{x}(t)
$$

The proportional and integral term can be included into the state vector in the common way defining the auxiliary state $z=\int_{0}^{t} y(t)$, i.e. $\dot{z}(t)=y(t)=C x(t)$. Then the closed-loop system for PI part of the controller is

$$
\dot{x}_{n}=\left[\begin{array}{c}
\dot{x} \\
\dot{z}
\end{array}\right]=\left[\begin{array}{cc}
A+\delta A & 0 \\
C & 0
\end{array}\right]\left[\begin{array}{l}
x \\
z
\end{array}\right]+\left[\begin{array}{c}
B+\delta B \\
0
\end{array}\right] u(t)
$$


and

$$
u(t)=F C x(t)+F_{d} C_{d} \dot{x}(t)
$$

where $F C x(t)$ and $F_{d} C_{d} \dot{x}(t)$ correspond respectively to the PI and D term of PID controller. The resulting closed loop system with PID controller (17) is then

$$
\dot{x}_{n}(t)=A_{C}(\alpha) x_{n}(t)+B(\alpha)\left[\begin{array}{ll}
F_{d} C_{d} & 0
\end{array}\right] \dot{x}_{n}(t)
$$

where the PI controller term is included in $A_{C}(\alpha)$. (For brevity we omit the argument $t$.) To simplify the denotation, in the following we consider PD controller (which is equivalent to the assumption, that the I term of PID controller has been already included into the system dynamics in the above outlined way) and the closed loop is described by

$$
\dot{x}(t)=A_{C}(\alpha) x(t)+B(\alpha) F_{d} C_{d} \dot{x}(t)
$$

Let us consider the following performance index

$$
J_{S}=\int_{0}^{\infty}\left[\begin{array}{ll}
x & \dot{x}
\end{array}\right]^{T}\left[\begin{array}{cc}
Q+C^{T} F^{T} R F C & 0 \\
0 & S
\end{array}\right]\left[\begin{array}{c}
x \\
\dot{x}
\end{array}\right] d t
$$

which formally corresponds to (6). Then for Lyapunov function (9) we have the necessary and sufficient condition for robust stability with guaranteed cost in the form (14), i.e. for continuous time system:

$$
\left[\begin{array}{ll}
x & \dot{x}
\end{array}\right]^{T}\left[\begin{array}{cc}
Q+C^{T} F^{T} R F C & P(\alpha) \\
P(\alpha) & S
\end{array}\right]\left[\begin{array}{c}
x \\
\dot{x}
\end{array}\right]<0 .
$$

The main result on robust PID control stabilization is summarized in the next theorem

Theorem 6. Consider a continuous uncertain linear system (1), (2) with PID controller (17) and cost function (21). The following statements are equivalent:

- Closed loop system (19) is robustly D-stable with PDLF (9) and guaranteed cost with respect to cost function (21):

$$
J \leq J_{0}=x^{T}(0) P(\alpha) x(0) .
$$

- There exist matrices $P(\alpha)>0$ defined by (9), and $H, G, \quad F$ and $F_{d}$ of the respective dimensions such that

$$
\left[\begin{array}{c}
A_{C i}^{T} H^{T}+H A_{C i}+Q+C^{T} F^{T} R F C \\
P_{i}-M_{d i}^{T} H+G^{T} A_{C i} \\
* \\
-M_{d i}^{T} G-G^{T} M_{d i}+S
\end{array}\right]<0
$$

$A_{C i}=\left(A_{i}+B_{i} F C\right)$ denotes the $i-$ th closed loop system vertex, $M_{d i}$ includes the derivative part of the PID controller: $M_{d i}=I-B_{i} F_{d} C_{d}$.

Proof. Owing to (20) for any matrices $H$ and $G$ :

$$
\begin{aligned}
& \left(-x^{T} H-\dot{x}^{T} G^{T}\right)\left(\dot{x}-A_{C}(\alpha) x-B(\alpha) F_{d} C_{d} \dot{x}\right)+ \\
& +\left(\dot{x}-A_{C}(\alpha) x-B(\alpha) F_{d} C_{d} \dot{x}\right)^{T}\left(H^{T} x-G \dot{x}\right)=0
\end{aligned}
$$

Summing up the 1.h.s of (24) and (22) and taking into consideration linearity w.r.t. $\alpha$ we get condition (23). Theorem 6 provides the robust stability condition for the linear uncertain system with PID controller. Notice that the derivative term does not appear in the matrix inversion and allows including the uncertainty in control matrix $B$ into the stability condition. 
Considering PID control design, there are unknown matrices $H, G, F$ and $F_{d}$ to be solved from (23). (Recall that $A_{C i}=\left(A_{i}+B_{i} F C\right), M_{d i}=I-B_{i} F_{d} C_{d}$.) Then the inequality (23) is not LMI, to cope with the respective unknown matrices products the linearizing approach using (13) has been adopted and the PID iterative control design algorithm based on LMI ( $4 \times 4$ matrix) has been proposed. The resulting closed loop system with PD controller is

$$
\dot{x}(t)=\left(I-B_{i} F_{d} C_{d}\right)^{-1}\left(A_{i}+B_{i} F C\right) x(t), i=1, \ldots, N
$$

The extension of the proposed algorithm to decentralized control design is straightforward since the respective $F$ and $F_{d}$ matrices are assumed as being of the prescribed structure, therefore it is enough to prescribe the decentralized structure for both matrices.

\section{Examples}

In this section the major contribution of the proposed approach: design of robust derivative feedback is illustrated on the examples. The results obtained using the proposed new iterative algorithm based on (23) to design the PD controller are provided and discussed. The impact of matrix $S$ choice is studied as well.

We consider affine models of uncertain system (1), (2) with symmetric uncertainty domain:

$$
\underline{\varepsilon}_{j}=-q, \overline{\varepsilon_{j}}=q
$$

Example 1.

Consider the uncertain system (1), (2) where:

$$
\begin{gathered}
A=\left[\begin{array}{ccc}
-4.365 & -0.6723 & -0.3363 \\
7.0880 & -6.5570 & -4.6010 \\
-2.4100 & 7.5840 & -14.3100
\end{array}\right] \quad B=\left[\begin{array}{cc}
2.3740 & 0.7485 \\
1.3660 & 3.4440 \\
0.9461 & -9.6190
\end{array}\right] \\
C=C_{d}=\left[\begin{array}{lll}
0 & 1 & 0 \\
0 & 0 & 1
\end{array}\right]
\end{gathered}
$$

uncertainty parameter $q=1$; uncertainty matrices:

$$
\begin{gathered}
\tilde{A}_{1}=\left[\begin{array}{ccc}
-0.5608 & 0.8553 & 0.5892 \\
0.6698 & -1.3750 & -0.9909 \\
3.1917 & 1.7971 & -2.5887
\end{array}\right] \quad \tilde{B}_{1}=\left[\begin{array}{cc}
-0.1602 & -0.3521 \\
0.1162 & -2.4839 \\
-0.1106 & -4.6057
\end{array}\right] \\
\tilde{A}_{2}=\left[\begin{array}{ccc}
0.6698 & -1.3750 & -0.9909 \\
-2.8963 & -1.5292 & 10.5160 \\
-3.5777 & 2.8389 & 1.9087
\end{array}\right] \quad \tilde{B}_{2}=\left[\begin{array}{cc}
0.1562 & 0.1306 \\
-0.4958 & 4.0379 \\
-0.0306 & 0.8947
\end{array}\right]
\end{gathered}
$$

The uncertain system can be described by four vertices; the corresponding maximal eigenvalues in the vertices of open loop system are respectively:

$-4.0896 \pm 2.1956 \mathrm{i} ;-3.9243 ; \mathbf{1 . 5 0 1 4} ;-4.9595$

Notice that the open loop uncertain system is unstable (see third vertex). The stabilizing optimal PD controller has been designed. Optimality is considered in the sense of guaranteed cost w.r.t. cost function (21) with matrices $R=I_{2 x 2}, Q=0.001 * I_{3 \times 3}$. The results summarized in the Tab. 1 indicate the differences between results obtained for different values of $S$ 


\begin{tabular}{|l|l|l|}
\hline $\mathrm{S}$ & $\begin{array}{l}\text { F (proportional part) } \\
\text { Fd (derivative part) }\end{array}$ & $\begin{array}{l}\text { Max Eig } \\
\text { in vertices }\end{array}$ \\
\hline & & \\
\hline 1 e-6 *I & $-1.0567-0.5643$ & -4.8644 \\
& $-2.1825-1.4969$ & $\mathbf{- 2 . 4 0 7 4}$ \\
& $-0.3126-0.2243$ & $-3.8368 \pm 1.1165 \mathrm{i}$ \\
& -0.09670 .0330 & -4.7436 \\
\hline & & \\
\hline $0.1 * \mathrm{I}$ & $-1.0724-0.5818$ & -4.9546 \\
& $-2.1941-1.4642$ & $\mathbf{- 2 . 2 2 1 1}$ \\
& $-0.3227-0.2186$ & $-3.7823 \pm 1.4723 \mathrm{i}$ \\
& -0.09690 .0340 & -4.7751 \\
\hline
\end{tabular}

Table 1: Example 1, Example 2

Consider the uncertain system (1), (2) where:

$$
\begin{gathered}
A=\left[\begin{array}{cccc}
-2.9800 & 0.9300 & 0 & -0.0340 \\
-0.9900 & -0.2100 & 0.0350 & -0.0011 \\
0 & 0 & 0 & 1 \\
0.3900 & -5.5550 & 0 & -1.89
\end{array}\right] \\
\tilde{A}_{1}=\left[\begin{array}{cccc}
0 & 1.5 & 0 & 0 \\
0 & 0 & 0 & 0 \\
0 & 0 & 0 & 0 \\
0 & 0 & 0 & 0
\end{array}\right] \\
B=\left[\begin{array}{c}
-0.0320 \\
0 \\
0 \\
-1.6000
\end{array}\right], \tilde{B}_{1}=\left[\begin{array}{l}
0 \\
0 \\
0
\end{array}\right] \\
C=\left[\begin{array}{llll}
0 & 0 & 1 & 0 \\
0 & 0 & 0 & 1
\end{array}\right]
\end{gathered}
$$

The results are summarized in Tab. 2 for $R=1, Q=0.0005 * I_{4 \times 4}$ for various values of matrix $S$ in cost function. As indicated in Tab.2, increasing values of $S$ slow down the response (max.eig. $C L$ shifted to zero) as assumed.

\section{Conclusion}

The new robust PID controller design method is proposed for uncertain linear system based on LMI. The important feature of this PID design approach is that the derivative term appears in such form that enables to consider the model uncertainties. Since the structured feedback matrix is assumed, this approach is appropriate for decentralized PID control design. The guaranteed cost control is proposed with a new quadratic cost function including the derivative term for state vector as a tool to influence the overshoot and response rate. The obtained results are illustrated on the examples: to show the robust PID control design and the influence of the choice of matrix $S$ in the extended cost function.

Acknowledgment The work has been supported by Slovak Scientific Grant Agency, Grant N 1/3841/06. 


\begin{tabular}{|l|l|}
\hline$S=10 \mathrm{e}-8 * I_{4 \times 4}$ & \\
\hline$q_{\max }$ & 1.1 \\
\hline max.eig. $C L$ & -0.189 \\
\hline & \\
\hline$S=0.1^{*} I_{4 x 4}$ & \\
\hline$q_{\max }$ & 1.1 \\
\hline $\operatorname{max.eig.} C L$ & -0.1101 \\
\hline & \\
\hline$S=0.2^{*} I_{4 \times 4}$ & \\
\hline$q_{\max }$ & 1.1 \\
\hline max.eig. $C L$ & -0.0863 \\
\hline & \\
\hline$S=0.29 * I_{4 x 4}$ & \\
\hline$q_{\max }$ & 1.02 \\
\hline max.eig. $C L$ & -0.0590 \\
\hline
\end{tabular}

Table 2: Comparison for various $S$ - Example 2

\section{References}

[1] Blondel, V. and J.N. Tsitsiklis (1997). NP-hardness of some linear control design problems. SIAM J. Control Optim., 35, pp.2118-2127.

[2] Boyd, S., L. El Ghaoui, E. Feron and V. Balakrishnan (1994). Linear matrix inequalities in system and control theory. SIAM Studies in Applied mathematics, Philadelphia.

[3] Han, J. and R.E. Skelton (2003). An LMI optimization approach for structured linear controllers. In: Proc. $42^{\text {nd }}$ IEEE CDC, Hawaii, USA, pp. 5143-5148.

[4] Henrion, D., D. Arzelier and D. Peaucelle (2002). Positive polynomial matrices and improved LMI robustness conditions. In: $15^{\text {th }}$ IFAC World Congress, Barcelona, Spain.

[5] deOliveira, M.C., J. Bernussou and J.C. Geromel (1999). A new discrete-time robust stability condition. Systems and Control Letters, 37, pp. 261-265.

[6] deOliveira, M.C., J.F. Camino and R.E. Skelton (2000). A convexifying algorithm for the design of structured linear controllers. In: Proc. $39^{\text {nd }}$ IEEE CDC, Sydney, Australia, pp. 2781-2786.

[7] Ge, Ming, Min-Sen Chiu, Qing-Guo Wang (2002). Robust PID controller design via LMI approach. Journal of Process Control, 12, pp.3-13.

[8] Gyurkovics, E., Takacs, T.( 2000): Stabilisation of discrete-time interconnected systems under control constraints. IEE Proceedings - Control Theory and Applications, 147, No. 2, 137-144

[9] Peaucelle, D., D. Arzelier, O. Bachelier and J. Bernussou (2000). A new robust $D$-stability condition for real convex polytopic uncertainty. Systems and Control Letters, 40, pp. 21-30.

[10] Rosinová, D., V. Veselý and V. Kučera (2003). A necessary and sufficient condition for static output feedback stabilizability of linear discrete-time systems. Kybernetika, 39, pp. 447-459.

[11] Rosinová, D. and V. Veselý (2003). Robust output feedback design of discrete-time systems - linear matrix inequality methods. In: $2^{\text {th }}$ IFAC Conf. CSD'03 (CD-ROM), Bratislava, Slovakia. 
[12] Skelton, R.E., T. Iwasaki and K. Grigoriadis (1998). A Unified Algebraic Approach to Linear Control Design, Taylor and Francis.

[13] Veselý, V. (2003). Robust output feedback synthesis: LMI Approach. In: $2^{\text {th }}$ IFAC Conference CSD’03 (CD-ROM), Bratislava, Slovakia.

[14] Zheng Feng, Qing-Guo Wang, Tong Heng Lee (2002). On the design of multivariable PID controllers via LMI approach. Automatica, 38, pp.517-526.

\author{
Danica Rosinová and Vojtech Veselý \\ Slovak University of Technology \\ Institute for Control and Industrial Informatics \\ Ilkovičova 381219 Bratislava, Slovakia \\ \{danica.rosinova; vojtech.vesely\}@stuba.sk \\ Received: December 24, 2006
}

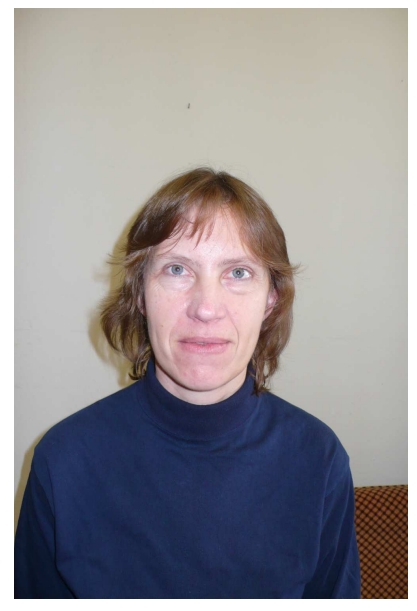

Danica Rosinová was born in Bratislava, Slovak Republic in 1961. She received MSc. and $\mathrm{PhD}$ from Slovak University of Technology in 1985 and 1996. Since 1985 she has been with the Department of Automatic Control Systems, now Institute for Control and Industrial Informatics at the Faculty of Electrical Engineering and Information Technology STU Bratislava. Since 2006 she has been Associate Professor. Her research interests concentrate on robust control, large scale systems theory and optimization. students.

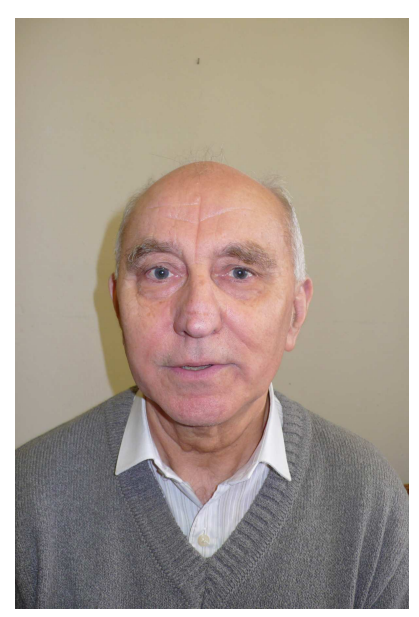

Vojtech Veselý was born in 1940. Since 1964 he has worked at the Department of Automatic Control Systems at the Faculty of Electrical Engineering and Information Technology, Slovak University of Technology in Bratislava. Since 1986 he has been Full Professor. His research interests include the areas of power system control, robust control, decentralized control of large-scale systems, proces control and optimization. He is author and coauthor of more then 270 scientific and technical papers, he successful supervised up today $19 \mathrm{PhD}$ 